\title{
Estudo epidemiológico das fraturas de coluna em centro de referência para patologia espinhal no Paraná
}

\author{
Matheus Lemos Vieira da Cunha1, Marcelo Lemos Vieira da Cunha², \\ Denildo César Amaral Veríssimo ${ }^{3}$, Roberta Rehder ${ }^{3}$, Luis Alencar Biurrum Borba ${ }^{4}$ \\ Departamento de Neurocirurgia do Hospital Universitário Evangélico de Curitiba, Curitiba, PR, Brasil.
}

\section{RESUMO}

Objetivo: Realizar um estudo epidemiológico das fraturas de coluna ocorridas no período de março de 2007 até dezembro de 2011 em um centro de referência para patologia espinhal. Métodos: Análise retrospectiva dos prontuários de pacientes submetidos a tratamento neurocirúrgico para artrodese da coluna vertebral por causa de patologia traumática, realizada de março de 2007 até dezembro de 2011. Os dados foram compilados a partir do setor de arquivo médico do Hospital Universitário Evangélico de Curitiba (HUEC). Os elementos encontrados foram analisados observando-se as variáveis idades, sexo, o nível e a vértebra mais acometida. Resultados: Dos 104 pacientes submetidos a tratamento neurocirúrgico visando à artrodese por causa de fratura de coluna, houve uma predominância considerável do sexo masculino, com $73,07 \%$ (76). A média de idade na data do procedimento cirúrgico foi de 38,08 anos. A idade mínima registrada foi de12 anos e a máxima, de 73 anos. Houve predomínio das fraturas em coluna lombar de $59,61 \%$ (62) dos casos. O corpo de $L 1$ foi mais acometido $(30,76 \%)$. Conclusão: Há escassez de trabalhos epidemiológicos no que se refere à patologia traumática da coluna vertebral. A falta de dados em nosso país dificulta a elaboração de medidas preventivas que respeitem as características regionais, vislumbrando um melhor manejo dessa patologia que atinge, em sua maior parte, a população economicamente ativa, gerando prejuízos crescentes ao sistema de saúde.

\section{PALAVRAS-CHAVE}

Fraturas da coluna vertebral, epidemiologia, traumatismos da medula espinal.

\section{ABSTRACT}

Epidemiological study of spine fractures at a referral center for spinal disease in Parana Objective: To conduct an epidemiological study of spine fractures in the period from March 2007 until December 2011 in a referral center for spinal pathology. Methods: Retrospective analysis of patients undergoing neurosurgical treatment for arthrodesis of the spine due to traumatic pathology, held from March 2007 until December 2011. Data were compiled from the medical file of the sector Evangelical Hospital of Curitiba (HUEC). The items found were analyzed observing the variables age, sex, level and most affected vertebra. Results: Of 104 patients who underwent neurosurgical intervention aimed arthrodesis due spine fracture, there was a significant predominance in males, with $73.07 \%$ (76). The mean age at the time of surgery was 38.08 years. The minimum age recorded was 12 years and maximum 73 years. There was a predominance of fractures in the lumbar spine $59.61 \%$ (62) of cases. L1 body was the most affected (30.76\%). Conclusion: There is a shortage of epidemiological studies in relation to the pathology of traumatic spinal cord. The lack of data in our country hampers the development of preventive measures that respect the regional characteristics, envisioning a better management of this disease that affects, for the most part, economically active population causing increasing damage to the health system.

\section{KEYWORDS}

Spinal fractures, epidemiology, spinal cord injuries.

Acadêmico do curso de Medicina da Universidade do Vale do Itajaí (Univali), Vale do Itajaí, SC, Brasil.

Neurocirurgião do Hospital Regional do Oeste, Chapecó, SC, Brasil.

3 Médico(a)-residente em Neurocirurgia do Hospital Universitário Evangélico de Curitiba, Curitiba, PR, Brasil.

4 Chefe do Departamento de Neurocirurgia do Hospital Universitário Evangélico de Curitiba, Curitiba, PR, Brasil. 


\section{Introdução}

A coluna vertebral humana é constituída por 33 vértebras. É dividida em cervical (7), torácica (12), lombar (5), sacral (5) e coccígea (4). As duas últimas são fundidas e formam o sacro, sendo denominadas vértebras falsas ou fixas. Eventualmente a coluna vertebral pode ser formada por 32 ou 34 vértebras. ${ }^{1}$ Dentre suas funções, destacam-se as de mobilidade e sustentação do corpo humano.

O traumatismo da coluna vertebral com lesão irreversível da medula é agudo e inesperado, ocasionando alterações drásticas à vida do indivíduo acometido, gerando desastrosas consequências ao lesado, à família e à sociedade. A importância de trabalhos epidemiológicos sobre pacientes com lesão medular torna-se evidente quando observamos que a única forma de melhorar a qualidade de vida da população e diminuir os custos com esses pacientes é evitar que as complicações aconteçam. ${ }^{2}$

Por causa de sua instalação abrupta e inesperada, com potencial de causar sequelas como paraplegia e quadriplégica, e sua maior incidência em indivíduos no pico de sua produtividade, o trauma de coluna vertebral representa um problema mundial de saúde pública. ${ }^{3}$ A taxa de incidência varia de 15 a 40 pacientes por 1 milhão de habitantes, sendo mais prevalente nas áreas urbanas. Tais números seguem aumentando a cada década. ${ }^{4}$

A incidência/prevalência exata de lesão medular traumática no Brasil é desconhecida, pois essa condição não é sujeita à notificação e há poucos dados e trabalhos publicados a respeito da epidemiologia da lesão espinhal. Estima-se que ocorram a cada ano no país mais de 10 mil novos casos de lesão medular, sendo o trauma a causa predominante. ${ }^{2}$

Segundo Hasler et al., ${ }^{5}$ cerca de metade das lesões graves de coluna não é suspeita no atendimento pré-hospitalar. A identificação de lesões na coluna vertebral durante a avaliação inicial do trauma é um desafio, pois os pacientes muitas vezes têm um nível reduzido de consciência devido a outras lesões ou estão sob a influência de medicação sedativa e/ou analgésica. A detecção precoce desse tipo de lesão pelo atendimento de emergência é importante a fim de iniciar testes diagnósticos e tratamento para evitar lesões adicionais na medula espinhal.

Além disso, outro estudo ${ }^{3}$ relatou que paciente com fratura vertebral tem o risco de novas fraturas de coluna aumentado em até 25 vezes se houver queda na densidade mineral óssea decorrente das lesões.

\section{Materiais e métodos}

Foi realizada uma análise retrospectiva de prontuários dos pacientes submetidos a tratamento neurocirúr- gico de artrodese para fusão espinhal a partir de março de 2007 até dezembro de 2011. Os dados foram compilados a partir do setor de arquivo médico do Hospital Universitário Evangélico de Curitiba (HUEC). Dos 335 pacientes selecionados, foram considerados apenas os que realizaram o procedimento por causa de fratura traumática da coluna, totalizando 107 pacientes. Desses, três foram excluídos por falta de dados para pesquisa: um deles por não constar a especificação do nível da vértebra fraturada e os outros dois por não terem a idade dos pacientes quando do ato cirúrgico.

Os dados encontrados foram analisados observando-se as variáveis idade, sexo, topografia da fratura (cervical, torácica e lombar) e a vértebra mais acometida.

As referências literárias foram obtidas por meio de pesquisa eletrônica no PubMed (www.pubmed.org) mediante procura dos termos: "spinal fracture", "epidemiological of spinal cord injury", "spinal cord injury", sem restrições. Os dados considerados pertinentes serão discutidos e comparados com os dados levantados.

\section{Resultados}

De março de 2007 a dezembro de 2011, selecionamos 104 pacientes submetidos a tratamento neurocirúrgico para artrodese espinhal por causa de fratura originada por trauma. Desses, $73,07 \%$ (76) eram do sexo masculino e $26,92 \%$ (28), do sexo feminino. A média de idade na data do procedimento cirúrgico foi de 38,08 anos. A idade mínima registrada foi em um paciente do sexo masculino com 12 anos. A idade máxima foi em um paciente do sexo masculino com 73 anos.

O maior número de cirurgias ocorreu quando os pacientes encontravam-se na terceira década de vida: 27,01\% (29) dos casos (Figura 1).

Da população total considerada no estudo, houve predomínio das fraturas em coluna lombar - 59,61\% (62) dos casos -, sendo o nível de L1 - 30,76\% (32) o de maior incidência (Figura 2). Dos pacientes que fraturaram a vértebra $L 1,56,25 \%$ (18) eram do sexo masculino e 43,75\% (14), do sexo feminino. A média de idade foi de 40,12 anos, e a média entre os homens foi de 34,94 anos e, entre as mulheres, de 46,78 anos.

O segundo nível da coluna vertebral mais acometido foi o torácico, com $26,92 \%$ (28) dos casos estudados. Já em relação à região cervical, obtivemos apenas $13,46 \%$ (13) das fraturas de coluna ocorridas de março de 2007 até dezembro de 2011 no HUEC.

Dos 76 casos registrados na população masculina, a idade média foi de 35,28 anos, com amplitude de 12 a 73 anos. As fraturas em vértebras lombares foram maioria: 47,36\% (36). Dessas, 47,22\% (17) foram em L1 (Figura 3). 


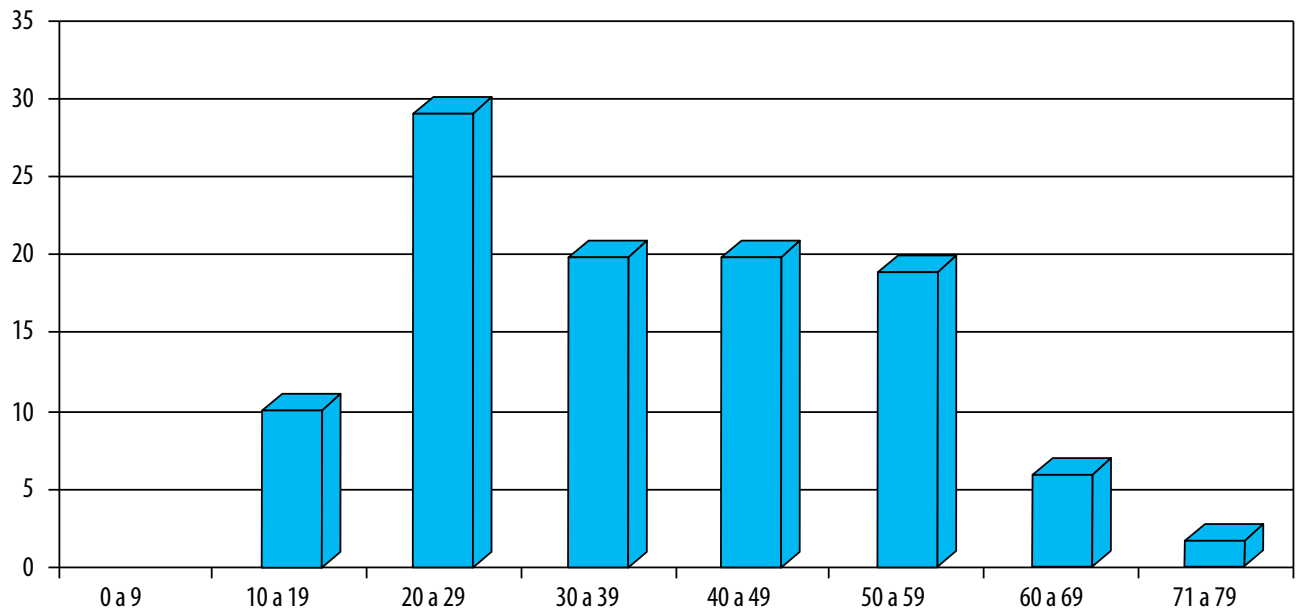

Figura 1 - Incidência das fraturas por décadas.

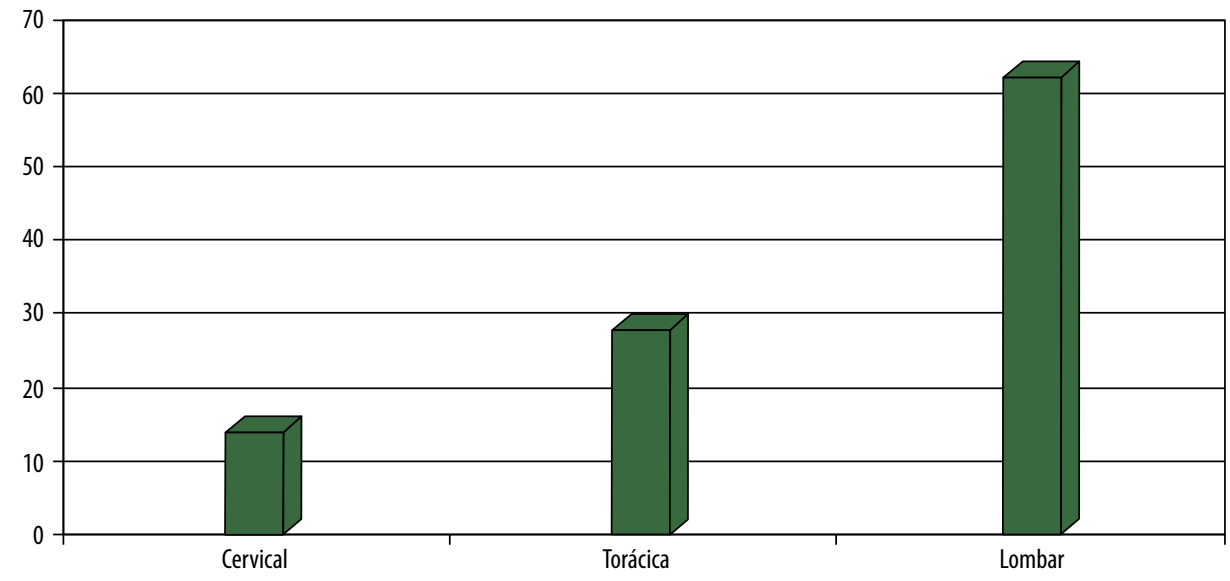

Figura 2 - Nível espinhal mais acometido.

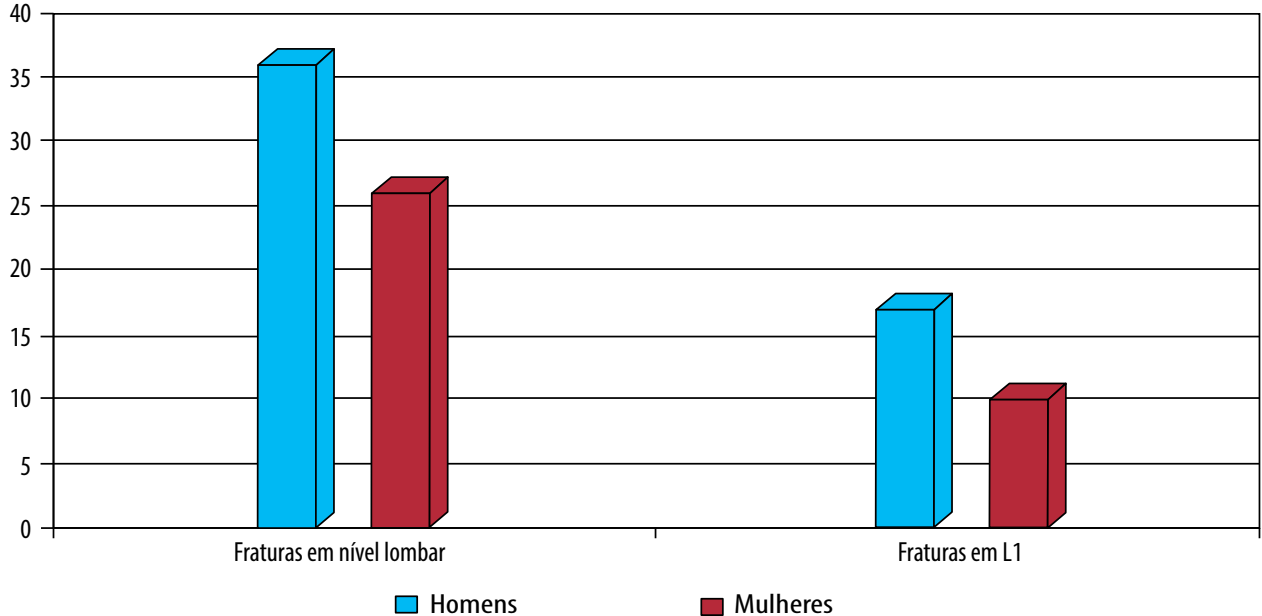

Figura 3 - Vértebra mais acometida entre homens e mulheres. 
Já com as 28 mulheres selecionadas para o estudo, obtivemos a média de idade de 45,67 anos, com uma amplitude de 20 a 62 . Assim como nos homens, a maioria das fraturas foi no nível lombar: $92,85 \%$ (26), sendo $53,84 \%$ (14) no nível de L1.

\section{Discussão}

A epidemiologia de determinada moléstia forma a base do sistema de saúde de qualquer país. Em nosso país ainda são escassos os estudos a respeito de fraturas de coluna, talvez pelo fato da grande extensão do país e/ou pela dificuldade na obtenção de dados.

Lesões traumáticas são os principais contribuintes para a morte e morbidade na população, sobretudo a economicamente ativa. Um dos primeiros passos para evitar que isso ocorra é a coleta e a análise de dados a fim de auxiliar na identificação dos fatores de riscos para a população. ${ }^{6}$

Em um dos estudos analisados, ${ }^{3}$ verificou-se que, em 2004, o Sistema Único de Saúde (SUS) registrou 15.700 internações por lesões medulares, com 505 mortes por fraturas de coluna, o que geralmente significa prolongadas permanências hospitalares, além de elevados gastos aos cofres públicos. Assim, o conhecimento sobre incidência, prevalência e consequências clínicas é essencial para o planejamento do tratamento e cuidados para esse grupo de pacientes nos hospitais.

Na série de Macciocchi et al., ${ }^{7}$ 34\% daqueles que tiveram uma lesão traumática da medula também tiveram um traumatismo craniano leve, e $26 \%$ tinham uma lesão grave na cabeça. Ou seja, indivíduos que sofrem acidente de trânsito e quedas correm um risco aumentado de sofrer, concomitantemente, um traumatismo craniano.

Lesão da medula espinhal resulta em consequências físicas, psicológicas, sociais e econômicas. Assim, é de extrema importância a identificação epidemiológica, demográfica e patológica das lesões da medula espinhal para identificar grupos de alto risco, tornando-se possível tomar as devidas providências e evitar essa forma de injúria. ${ }^{8}$

Comparando os dados deste estudo com os demais analisados, os resultados de nosso levantamento corroboram a predominância do sexo masculino nesse tipo de patologia traumática. É o que podemos verificar nos estudos de Brito et al., ${ }^{3}$ Santos et al. ${ }^{4}$ e Hasler et al. ${ }^{5}$ Todos apresentam mais de $60 \%$ de prevalência masculina nos casos de trauma na coluna vertebral.

No trabalho de Santos et al., ${ }^{4}$ a proporção entre homens e mulheres foi de 7,35:1, enquanto o nosso obteve um índice de 2,71:1. Embora a prevalência masculina tenha sido mantida, chama a atenção a maior despro- porção entre homens e mulheres daquele estudo. Uma das explicações para esse destoante pode residir na amostra maior do primeiro - 217 pacientes.

Em um estudo com 942 pacientes irlandeses com fratura de coluna vertebral e/ou lesão da medula por trauma, verificou-se que os homens são mais expostos a riscos em todas as etiologias das lesões espinhais. ${ }^{9} \mathrm{Em}$ todos os estudos por nós analisados, os homens foram mais prevalentes.

Levando em consideração apenas a média de idade, a maioria dos estudos ${ }^{3-5}$ aponta a quarta década de vida como a de maior incidência em traumas na coluna vertebral.

O trabalho de Tator et al. ${ }^{10}$ apresentou como resultado $64,5 \%$ de lesões em vértebras cervicais, enquanto em outra série analisada ${ }^{2}$ o segmento da coluna significativamente mais comprometido foi o torácico, com $37,9 \%$ dos casos. Nossa série destoou dessas estatísticas, apresentando 59,61\% das lesões em vértebras lombares, sendo a primeira vértebra lombar (L1) a mais acometida. Das séries analisadas, apenas a de Pirouzmand ${ }^{6}$ atingiu resultados parecidos com os nossos, pois também obteve a região lombar como a mais acometida em $50 \%$ dos casos.

Dentre as causas de trauma na coluna vertebral, a queda de nível é descrita como um dos principais fatores de risco., ${ }^{3,6,11,12}$ Segundo Campos et al., ${ }^{2}$ as causas principais de traumatismo da coluna vertebral foram predominantemente ocasionadas pelas quedas, com $63 \%$, seguidas dos acidentes automobilísticos: $25 \%$. Em menor frequência, encontramos os ferimentos por arma de fogo (em torno de 7\%), os mergulhos em águas rasas (3\%) e as agressões $(2 \%)$.

Já na série de Tuğcu et al. ${ }^{8}$ foram encontradas como maior prevalência as colisões de automóveis, seguidas de queda de nível e ferimento por arma de fogo. Na África do Sul, trauma por violência é uma causa frequente de lesões da medula espinhal, responsável por $61 \%-62 \%$ dos casos. ${ }^{13}$

Acreditamos que a variação das principais causas de trauma nas séries estudadas é devida às diferenças geográficas, sociais e culturais dos países. Também podem ter ocorrido essas disparidades pela forma com que foi realizada a compilação desses dados - ou mesmo pela escassez deles - para realização da avaliação epidemiológica.

Segundo Hartkopp et al., ${ }^{14}$ a expectativa de vida entre os indivíduos com lesões medulares manteve-se menor do que na população normal, mesmo com tratamento médico ideal. A pesquisa incluiu um total de 888 indivíduos que haviam sobrevivido à lesão e ao tratamento primário e foram reabilitados. No final do seguimento, 236 tinham falecido. As causas mais comuns de morte foram: doenças pulmonares, especialmente pneumonia, suicídio e doença isquêmica do coração. 


\section{Conclusão}

A falta de dados em nosso país referentes à epidemiologia do trauma espinhal dificulta a elaboração de medidas preventivas, bem como de maior suporte ao acometido por trauma raquimedular. Em comparação a trabalhos realizados no exterior, podemos verificar semelhanças e diferenças nos dados, o que pode conotar diferenças regionais, as quais devem ser levadas em consideração quando da elaboração de medidas de saúde pública.

Esta publicação fornece um estudo atualizado sobre a incidência de fratura de coluna voltada à comunidade neurocirúrgica numa área geográfica bem definida. Acreditamos que o conhecimento regionalizado permite uma melhor assistência à população local, com o desenvolvimento de centros especializados em determinada patologia objetivando melhores resultados funcionais e, consequentemente, economia aos cofres públicos.

\section{Referências}

1. Gross CM, editor. Anatomia. $29^{a}$ ed. Rio de Janeiro: Guanabara Koogan; 1988.

2. Campos MF, Ribeiro AT, Listik S, Pereira CAB, Andrade Sobrinho J, Rapoport A. Epidemiologia do traumatismo da coluna vertebral. Rev Col Bras Cir. 2008;35(2):88-93.

3. Brito LMO, Chein MBC, Marinho SC, Duarte TB. Epidemiological evaluation of victims of spinal cord injury. Rev Col Bras Cir. 2011;38(5):304-9.

4. Santos EA, Filho WJ, Possatti LL, Bittencourt LR, Fontoura EA, Botelho RV. Epidemiology of severe cervical spinal trauma in the North area of São Paulo City: a 10-year prospective study. J Neurosurg Spine. 2009;11(1):34-41.
5. Hasler RM, Exadaktylos AK, Bouamra O, Benneker LM, Clancy M, Sieber R, et al. Epidemiology and predictors of spinal injury in adult major trauma patients: European cohort study. Eur Spine J. 2011;20(12):2174-80.

6. Pirouzmand F. Epidemiological trends of spine and spinal cord injuries in the largest Canadian adult Trauma Center from 1986 to 2006. J Neurosurg Spine. 2010;12(2):131-40.

7. Macciocchi SN, Bowman B, Coker J, Apple D, Leslie D. Effect of co-morbid traumatic brain injury on functional outcome of persons with spinal cord injuries. Am J Phys Med Rehabil. 2004;83(1):22-6.

8. Tuğcu I, Tok F, Yılmaz B, Göktepe AS, Alaca R, Yazıcıoğlu $\mathrm{K}$, et al. Epidemiologic data of the patients with spinal cord injury: seven years' experience of a single center. Ulus Travma Acil Cerrahi Derg. 2011;17(6):533-8.

9. Lenehan B, Boran S, Street J, Higgins T, McCormack D, Poynton AR. Demographics of acute admissions to a National Spinal Injuries Unit. Eur Spine J. 2009;18(7):938-42.

10. Tator $\mathrm{CH}$, Fehlings MG, Thorpe K, Taylor W. Current use and timing of spinal surgery for management of acute spinal surgery for management of acute spinal cord injury in North America: results of a retrospective multicenter study. $J$ Neurosurg. 1999;91(Suppl 1):12-8.

11. Pickett W, Simpson K, Walker J, Brison RJ. Traumatic spinal cord injury in Ontario, Canada. J Trauma. 2003;55(6):1070-6.

12. Nobunaga Al, Go BK, Karunas RB. Recent demographic and injury trends in people served by the Model Spinal Cord Injury Care Systems. Arch Phys Med Rehabil. 1999;80(11):1372-82.

13. Hagen EM, Rekand T, Gilhus NE, Grønning M. Traumatic spinal cord injuries: incidence, mechanisms and course. Tidsskr Nor Laegeforen. 2012;132(7):831-7.

14. Hartkopp A, Brønnum-Hansen H, Seidenschnur AM, Biering-Sørensen F. Survival and cause of death after traumatic spinal cord injury. A long-term epidemiological survey from Denmark. Spinal Cord. 1997;35(2):76-85.

Endereço para correspondência

Marcelo Lemos Vieira da Cunha

Rua Rui Barbosa, 93 E, ap. 501, Centro

89801-040 - Chapecó, SC, Brasil

E-mail:marcelolvc@yahoo.com.br 